\title{
Silymarin inhibits proliferation of human breast cancer cells via regulation of the MAPK signaling pathway and induction of apoptosis
}

\author{
SUNG-HYUN KIM, GANG-SIK CHOO, EUN-SEON YOO, JOONG-SEOK WOO, JAE-HAN LEE, \\ SO-HEE HAN, SOO-HYUN JUNG, HYEONG-JIN KIM and JI-YOUN JUNG \\ Department of Companion and Laboratory Animal Science, Kongju National \\ University, Yesan, Chungcheongnam 340-702, Republic of Korea
}

Received November 19, 2019; Accepted February 16, 2021

DOI: $10.3892 / \mathrm{ol} .2021 .12753$

\begin{abstract}
Silymarin is a purified mixture of four isomeric flavonoids extracted from the seeds and fruit of the milk thistle plant, Silybum marianus (L.). Silymarin exhibits a wide variety of biological effects and is commonly used in traditional medicine. Therefore, the anticancer effects of silymarin on human breast cancer cells were investigated to determine its pharmacological mechanisms in vitro and in vivo. The viability and proliferation of MDA-MB- 231 and MCF-7 breast cancer cells were investigated using MTT and wound healing assays. Silymarin decreased the viability and proliferation of MDA-MB-231 and MCF-7 cells in a concentration-dependent manner. The number of apoptotic bodies, as shown by DAPI staining, was increased in a concentration-dependent manner, indicating that silymarin induces apoptosis. Additionally, changes in the expression levels of apoptosis-related proteins were demonstrated in human breast cancer cells using western blotting. Silymarin increased the levels of Bax, cleaved poly-ADP ribose polymerase, cleaved caspase- 9 and phosphorylated (p-)JNK, and decreased the levels of Bcl-2, p-P38 and p-ERK1/2. Furthermore, the inhibitory effects of silymarin on MCF-7 tumor growth were investigated. In mice treated with silymarin for 3 weeks (25 and $50 \mathrm{mg} / \mathrm{kg}$ ), MCF-7 tumor growth was inhibited without organ toxicity. In MCF-7 tumors, silymarin induced apoptosis and decreased p-ERK1/2 levels, as assessed using a TUNEL assay and immunohistochemistry. These results indicated that silymarin inhibited breast cancer cell proliferation both in vitro and in vivo by modulating the MAPK signaling pathway. Therefore, silymarin may potentially be used as a chemo-preventive or therapeutic agent.
\end{abstract}

Correspondence to: Professor Ji-Youn Jung, Department of Companion and Laboratory Animal Science, Kongju National University, 54, Daehak-ro, Yesan-eup, Yesan, Chungcheongnam 340-702, Republic of Korea

E-mail: wangza@kongju.ac.kr

Key words: silymarin, apoptosis, breast cancer, MAPK signaling pathway, tumor

\section{Introduction}

The incidence of breast cancer in China and other parts of Asia is rapidly increasing. Breast cancer is also the second leading cause of cancer-related death in females in the United States (1). A number of methods are currently used to treat the breast cancer, including chemotherapeutic agents, surgery and radiotherapy, however, these methods do not improve the incidence of prognosis, and survival rates. Breast cancer can be categorized according to the level of estrogen receptor (ER) expression on the cancer cells, ER-positive and ER-negative. The ER is expressed in $\sim 60 \%$ of all breast cancer cases. ER-positive breast cancer generally has a good prognosis and patients exhibit a favorable response to antiestrogen therapy. However ER-negative breast cancers are more aggressive and unresponsive to antiestrogen therapy $(2,3)$. Therefore, an exploration of novel therapeutic agents for breast cancer treatment and prevention is required.

Flavonoids are synthesized by plants, and are a group of polyphenolic compounds with similar structures. The flavonoids are categorized into subclasses, including the anthocyanidins, flavanols, flavanones, flavanols, flavones and isoflavones. Flavonoids carry out a number of beneficial roles in the human body, including antioxidant, anti-inflammatory, and anti-carcinogenic effects (4). Silymarin that is a purified mixture of four isomeric flavonoid, and is extracted from the seeds and fruit of the milk thistle plant that commonly known as Silybum marianus (L.) Gaertn. The plant contains $\sim 65-80 \%$ silymarin flavonolignans with small amounts of flavonoids and 20-35\% fatty acids and other polyphenolic compounds. The major component of the silymarin complex is silybin, which is synonymous with silibinin (5). Several clinical trials have shown that this mixture exhibits significant hepatoprotective effects (6). More recently, silymarin was reported to exhibit various biological effects, including antioxidant (7) anti-inflammatory (8) and anti-cancer activities (9). In addition, silymarin may modulate the balance between pro-survival and pro-apoptotic signals by interfering with the expression of cell cycle- and apoptosis-regulating proteins, respectively (10).

Apoptosis and necrosis are known to intercellular mechanisms of cell death. Apoptosis is a form of programmed cell 
death, while necrosis is a form of cellular injury that induces an inflammatory tissue response to the external leakage of intracellular materials (11). Apoptosis is induced by the expression of various proteins, including the Bcl-2 family and mitogen-activated protein kinase (MAPK) pathway proteins in response to physical or chemical stimulation of DNA (12). The Bcl-2 family is known to inhibit the genesis and progression of cancer via apoptosis, and is classified into the pro-apoptotic proteins and anti-apoptotic proteins (13). The pro-apoptotic proteins include Bax which induces apoptosis by permeating the outer mitochondrial membrane, while the anti-apoptotic proteins, which include $\mathrm{Bcl}-2$ and $\mathrm{Bcl}-\mathrm{xl}$, inhibit apoptosis by preserving the outer mitochondrial membrane (14). Also, the actions of caspase- 9 have a direct impact on the mitochondria as well as upstream effectors of intrinsic apoptosis (15). The MAPKs comprise extracellular signal-regulated protein kinase (ERK1/2), P38 MAPK, and c-Jun N-terminal kinase/stress-activated protein kinase (JNK/SAPK), which regulate biological activities such as cell signaling transmission and play an important role in cell death and proliferation (16).

These study was to determine, in MDA-MB-231 and MCF-7 human breast cells, the inductive effects of silymarin on apoptosis in vitro, and to explore the mechanisms of MAPK signaling transmission. In addition, the effects of silymarin on in vivo tumor growth were investigated.

\section{Materials and methods}

Reagents and antibodies. Silymarin was purchased from Sigma-Aldrich; Merck KGaA (cat. no. S0292). RPMI-1640, fetal bovine serum (FBS) and penicillin-streptomycin were purchased from Welgene, Inc. 3-(4,5-dimethylthiazol-2-yl)2,5-diphenyltetrazolium bromide (MTT), cell lysis buffer, 4', 6-diamidino-2-phenylindole (DAPI) and dimethyl sulfoxide (DMSO) were also purchased from Sigma-Aldrich; Merck KGaA. Anti- $\beta$-actin, anti-Bax, anti-Bcl-2, anti-caspase-9, anti-poly [ADP-ribose] polymerase (PARP), anti-phosphorylated (p-)P38, anti-p-JNK, anti-p-ERK1/2, anti-p38, anti-JNK, anti-ERK1/2 and goat anti-rabbit IgG antibodies were purchased from Cell signaling Technology, Inc.

Cell line and culture. The human MDA-MB-231 and MCF-7 breast cancer cell lines were obtained from the Korean Cell Line Bank (Seoul, Korea). And cultured in RPMI-1640 medium, supplemented with $10 \%$ FBS and $1 \%$ penicillin-streptomycin. The cells were maintained at $37^{\circ} \mathrm{C}\left(5 \% \mathrm{CO}_{2}\right)$ in a humidified atmosphere. And the culture medium was replaced every two to three days.

Cell viability assay. Silymarin-induced changes in breast cancer cell viability were assessed using a MTT assay. MDA-MB-231 and MCF-7 cells were seeded into 96-well plate at a density of $2 \times 10^{4}$ cells $/ \mathrm{ml}(200 \mu \mathrm{l} /$ well). Following incubation for $24 \mathrm{~h}$ at $37^{\circ} \mathrm{C}\left(5 \% \mathrm{CO}_{2}\right)$, the cells were treated with silymarin $0,25,50,75,100,150$ and $200 \mu \mathrm{g} / \mathrm{ml}$ for $24 \mathrm{~h}$. We checked the concentrations that based on the previous experiment in the reference $(9,10)$. Silymarin was dissolved in alcohol and diluted in the media. After treatment, the medium was discarded, and $40 \mu \mathrm{l}$ MTT solution $(5 \mathrm{mg} / \mathrm{ml})$ was added prior to incubation for a further $2 \mathrm{~h}$. Subsequently, $100 \mu \mathrm{l}$ of DMSO was added to each well to dissolve the formazan crystals and the absorbance was recorded at $595 \mathrm{~nm}$ using a microplate reader (Bio-Rad, Laboratories, Inc). Untreated control cells were used as the comparative control.

Wound healing assay. MDA-MB-231 and MCF-7 cells were seeded in growth medium into culture diches and incubated for $24 \mathrm{~h}$. After confirming the formation of a complete monolayer, a uniform scratch was created in each dish using a sterile 1-ml pipette tip, and the cells were washed three times with PBS. The medium was replaced with $5 \%$ serum medium in the presence of absence of silymarin, and the cells were incubated for $24 \mathrm{~h}$. The wound closure rate was assessed at 0 and $24 \mathrm{~h}$ using images captured with a phase-contrast microscope (magnification, x200).

DAPI staining. DAPI staining was performed to detect the chromatin condensation and nuclear fragmentation, known characteristics of the apoptotic cells. MDA-MB-321 and MCF-7 cells were seeded into $60-\mathrm{mm}$ culture dishes and allowed to attach for $24 \mathrm{~h}$. The cells were then treated PBS or various concentrations of silymarin for $24 \mathrm{~h}$. After discarding the media, the cells were washed with PBS and incubated with $4 \%$ formalin and methanol for $15 \mathrm{~min}$ each at room temperature. DAPI solution was added and the cells were incubated at room temperature for $10 \mathrm{~min}$ under light protection. Chromatin condensation was observed under a fluorescence microscope (magnification, x200) and apoptotic bodies were counted three times in 100 randomly selected cells.

Western blot analysis. MDA-MB-231 and MCF-7 cells were treated with various concentrations of silymarin for $24 \mathrm{~h}$, and then the cells were harvested and lysed for $30 \mathrm{~min}$ on ice. A Bradford protein assay (Bio-Rad Laboratories, Inc.) was used to determine the concentration of each protein. The proteins were separated using 6-14\% SDS PAGE gels, and subsequently transferred onto nitrocellulose membranes. The membranes were blocked with blocking buffer 5\% non-fat dry milk in Tris-buffered saline with Tween-20 (TBS-T) for $1 \mathrm{~h}$ at room temperature, and then were further incubated with primary antibodies (diluted in blocking solution) overnight at $4^{\circ} \mathrm{C}$. The following primary antibodies were purchased from Cell Signaling Technology, Inc.: Anti- $\beta$-actin $(1: 1,000$; cat. no. 4967), anti-Bax (1:1,000; cat. no. 2772), anti-Bcl-2 (1:1,000; cat. no. 2876), anti-caspase-9 (1:1,000; cat. no. 9502), anti-PARP (1:1,000; cat. no. 9542), anti-p-P38 (1:1,000; cat. no. 4631), anti-p38 (1:1,000; cat. no. 9212), anti-p-JNK (1:1,000; cat. no. 4668), anti-JNK (1:1,000; cat. no. 9252), anti-p-ERK1/2 (1:1,000; cat. no. 9101), anti-ERK1/2 (1:1000; cat. no. 9102; Cell Signaling Technology, Inc.). After washing with TBS-T, the membranes were incubated with horseradish peroxidase (HRP)-conjugated, goat anti-rabbit $\mathrm{IgG}$ secondary antibodies (1:1,000; cat. no. 7074; Cell Signaling Technology, Inc.) for $1 \mathrm{~h}$ at room temperature. After further washing with TBS-T, the protein bands were visualized using enhanced chemiluminescence (ECL) detection reagents (Pierce; Thermo Fisher Scientific, Inc.) according to the manufacturer's instructions. For each band, the concentration was quantified using the ImageJ Launcher (version 1.48; NCBI) imaging program. 
Animals and in vivo xenograft tumor model. Male BALB/c nude mice (5-week-old, male, $20 \mathrm{~g}$ ) were purchased from Orient Bio Inc. (Gyeonggi-do, Korea). The mice were maintained under a $12 \mathrm{~h}$ light/dark cycle and housed at a controlled temperature $\left(23 \pm 3^{\circ} \mathrm{C}\right)$ and humidity $(40 \pm 10 \%)$ conditions. The mice were maintained in isolated and ventilated cages ( $\leq 3$ mice per cage) and allowed access to pelleted laboratory food and water ad libitum. MCF-7 cells were subcutaneously injected into the right flanks of the donor nude mice. After seven days established, MCF-7-cell tumors were detectable; when the tumors were palpable, the mice were randomly divided into 3 groups ( $n=5$ per group). Silymarin was dissolved in alcohol and diluted with distilled water (DW) and then orally administered 5 times per week at a dose of 25 or $50 \mathrm{mg} / \mathrm{kg}$ body weight, while the control group mice were administered the vehicle (distilled water) and the same amount of alcohol that used to dissolve the silymarin. Tumor weight and size were measured twice a week for a total of 21 days. We confirmed the previous experiments using silymarin to set the concentration of silymarin that administered to mice. The duration of experiment in 21 day was established by referring in the previous silymarin experiment of the reference. Tumor size should not exceed $1.5 \mathrm{~cm}$ per IACUC guideline, so we completed the experiment in 21 day to reduce the pain in according to the animal ethics (9). Tumor volume was measured as follows: volume $\left(\mathrm{mm}^{3}\right)=[0.5 \mathrm{x}(\mathrm{a}+\mathrm{b})]^{3}$, where a was the long axis, and $b$ the short axis of the tumor. At the end of the experimental period, the mice were sacrificed by cervical dislocation and the tumors were excised and weighted. A portion of the tumor was embedded in paraffin and used for TUNEL assays and immunohistochemical analyses. The animal experiments were conducted in accordance with the regulations of the Institutional Animal Care and Use Committee with the approval of the Ethics Committee of Kongju National University (Chungcheongnam-do, Korea; approval no. KNU_2018-6).

TUNEL assay. Tumor tissues were analyzed using TUNEL in situ apoptosis detection kit (Promega Corporation) according to the manufacturer's protocol. Briefly, paraffin-embedded sections $(5 \mu \mathrm{m})$ were deparaffinized and rehydrated, after which the sections were incubated with proteinase $\mathrm{K}$ for $15 \mathrm{~min}$ at room temperature. Equilibration buffer, biotinylated nucleotide mixture and recombinant terminal deoxynucleotidyl transferase (rTdT) were mixed, added to each slide, and allowed to react at $37^{\circ} \mathrm{C}$ for $1 \mathrm{~h}$. Then, $0.3 \%$ hydrogen peroxide in PBS was added and allowed to react for $5 \mathrm{~min}$. Streptavidin-HRP was then added to each slide, followed by 3,3'-diaminobenzidine tetrahydrochloride (DAB) solution, which was allowed to react for $10 \mathrm{~min}$. The slides were rinsed several times in deionized water and mounted. Brown-colored apoptotic bodies in the tumor sections from the control and silymarin-treated mice were counted using a light microscope (magnification, $\mathrm{x} 400$ ).

Immunohistochemistry. The paraffin-embedded sections were deparaffinized and rehydrated by sequential immersion in xylene and alcohol solutions. The sections were then incubated with anti-p-ERK1/2 (1:100) antibodies at $4^{\circ} \mathrm{C}$ overnight and subsequently incubated with a peroxidase-conjugated goat anti-rabbit antibody for $1 \mathrm{~h}$ at room temperature. The tumor sections were visualized using DAB solution. After mounting, the sections was observed using a light microscope (magnification, $\mathrm{x} 200)$.

Histological examination. The livers and kidneys of MCF-7-tumor xenograft mice were fixed in $10 \%$ neutral formalin buffer at room temperature and embedded in paraffin. The paraffin-embedded tissue were cut into 5 - $\mu \mathrm{m}$ sections and stained with hematoxylin and eosin. Histopathological changes were assessed using a light microscope (magnification, x200)

Statistical analysis. The experimental results are presented as the means \pm standard deviation (SD) or standard error (SE). Statistical analyses were conducted one-way ANOVA (SPSS Statistics 17.0; IBM Corp.) followed by Dunnett's t-tests. When determining the differences between the mean group values, $\mathrm{P}<0.05$ was considered to indicate a statistically significant difference.

\section{Results}

Effects of silymarin on the cell viability of breast cancer cells. A MTT assay was performed to evaluate the effects of silymarin on MDA-MB-231 and MCF-7 breast cancer cells. Cells were treated with $0,25,50,75,100,150$ or $200 \mu \mathrm{g} / \mathrm{ml}$ silymarin for $24 \mathrm{~h}$. The percentage of cell viability MDA-MB-231 cells were $114.7 \%$ at $25 \mu \mathrm{g} / \mathrm{ml}, 106.7 \%$ at $50 \mu \mathrm{g} / \mathrm{ml}, 92.2 \%$ at $75 \mu \mathrm{g} / \mathrm{ml}$, $75.1 \%$ at $100 \mu \mathrm{g} / \mathrm{ml}, 63.1 \%$ at $150 \mu \mathrm{g} / \mathrm{ml}$ and $51.5 \%$ at $200 \mu \mathrm{g} / \mathrm{ml}$ silymarin (Fig. 1A). The percentages of cell viability MCF-7 cells were $108.0 \%$ at $25 \mu \mathrm{g} / \mathrm{ml}, 90.2 \%$ at $50 \mu \mathrm{g} / \mathrm{ml}, 69.9 \%$ at $75 \mu \mathrm{g} / \mathrm{ml}, 49.5 \%$ at $100 \mu \mathrm{g} / \mathrm{ml}, 30.5 \%$ at $150 \mu \mathrm{g} / \mathrm{ml}$ and $26.1 \%$ at $200 \mu \mathrm{g} / \mathrm{ml}$ silymarin (Fig. 1B). These results suggest that silymarin has significantly effects the viability of MDA-MB-231 and MCF-7 human breast cancer cells. Also, MCF-7 cells were more sensitive to silymarin than MDA-MB-231 cells.

Effects of silymarin on the proliferation of breast cancer cells. Cancer cells can generate new tumors by infiltrating into distant tissues via the lymphatic ducts and blood, or by proliferating into the surrounding tissues (17). As proliferation is one of the primary characteristics of cancer cells, MDA-MB-231 and MCF-7 cells were treated with concentrations of silymarin that significantly impacted the cell viability (as determined by MTT assay), and proliferation was assessed using a wound-healing assay. We checked the survival rate through the screening that used the MTT assay. Based on these results, we set the concentrations at MDA-MB-231 and MCF-7. Following treatment with silymarin (MDA-MB-231 cells, 100 and $200 \mu \mathrm{g} / \mathrm{ml}$ and MCF-7 cells 50 and $100 \mu \mathrm{g} / \mathrm{ml}$ ), the proliferation of both breast cancer cells was inhibited in a concentration-dependent manner (Fig. 2A). In addition, the cells percentages at the $24 \mathrm{~h}$ time point were $100 \%$ (untreated), $50.6 \%(100 \mu \mathrm{g} / \mathrm{ml}))$, and $12.1 \%(200 \mu \mathrm{g} / \mathrm{ml})$ for MDA-MB-231 and $100 \%$ (untreated), $65.3 \%(50 \mu \mathrm{g} / \mathrm{ml})$, and $21.6 \%(100 \mu \mathrm{g} / \mathrm{ml})$ for MCF-7 cells, compared with those of the control group, indicating concentration-dependent inhibition of cellular proliferation (Fig. 2B). Accordingly, these findings indicate that silymarin exerts inhibitory effects on the proliferation of MDA-MB-231 and MCF-7 breast cancer cells. 
A

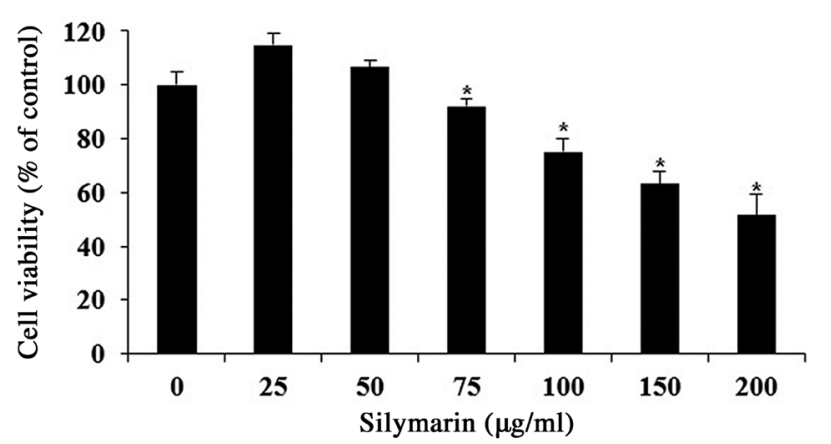

B

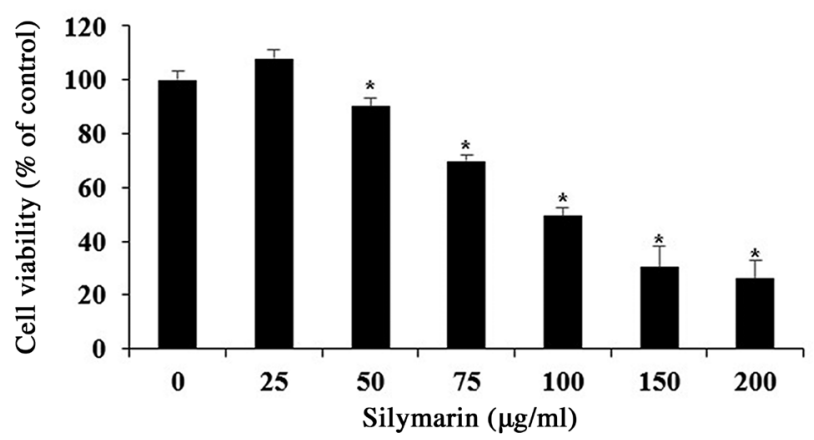

Figure 1. Effects of silymarin on the viability of breast cancer cells. (A) MDA-MB-231 and (B) MCF-7 cells were treated with silymarin for 24 h and cell viability was assessed using an MTT assay. The results are expressed as the percentages of cell viability compared with the non-treated control group, and presented as the mean $\pm \mathrm{SD}$ of three independent experiments. ${ }^{*} \mathrm{P}<0.05$ vs. untreated control $(0 \mu \mathrm{g} / \mathrm{ml}$ silymarin).

A
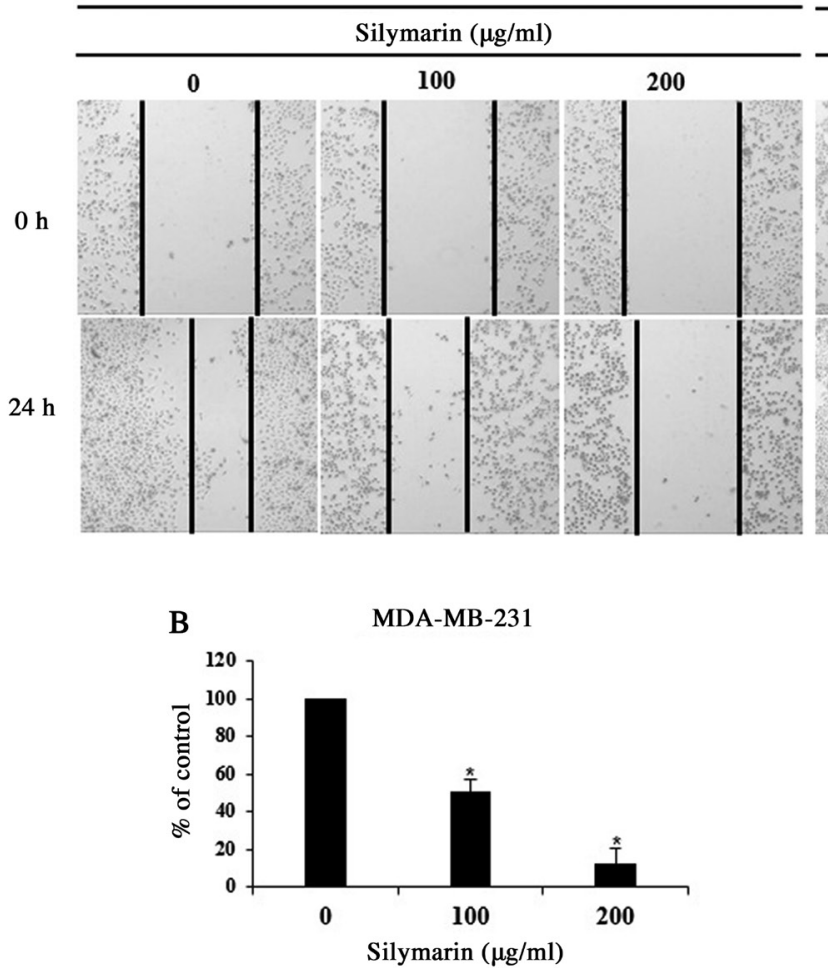

MCF-7

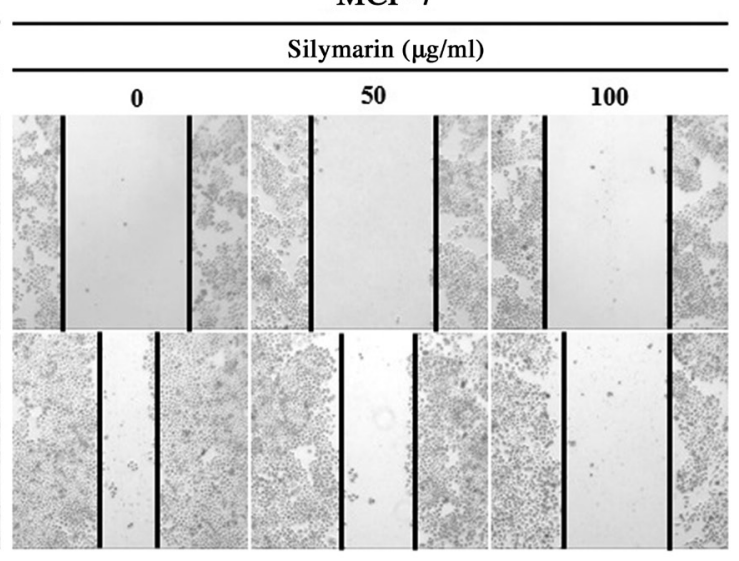

MCF-7

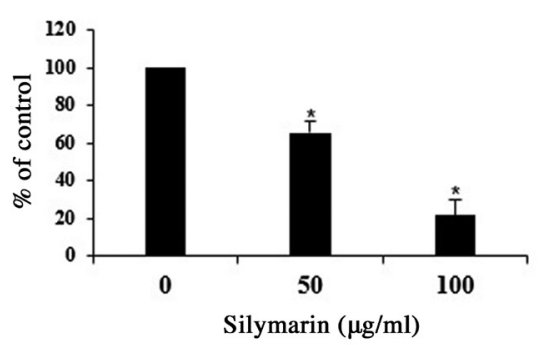

Figure 2. Effect of silymarin on the proliferation of breast cancer cells. MDA-MB-231 and MCF-7 cells were treated with the indicated concentrations of silymarin for $24 \mathrm{~h}$. (A) Wound healing assay. Magnification, x200. (B) Graphs showing the percentage of wound closure compared with the non-treated control group at $24 \mathrm{~h}$ after scratching. Data are presented as the mean $\pm \mathrm{SD}$ of three independent experiments. $\mathrm{P}<0.05 \mathrm{vs}$. untreated control ( $0 \mu \mathrm{g} / \mathrm{ml}$ silymarin).

Silymarin-induced morphological changes in breast cancer cells. To investigated whether the silymarin-induced effects on breast cancer cells viability resulted from the induction of apoptosis, chromatin condensation and morphological changes to the nucleus were observed using DAPI staining, which specifically interacts with DNA (11). DAPI-positive cells were considerably more likely to be found in silymarin-treated MDA-MB-231 and MCF-7 breast cancer cells than in the untreated control cells (Fig. 3A and B). To assess the degree of apoptosis, images of 100 cells from five random fields were captured using a fluorescence microscope (magnification, $\mathrm{x} 200$ ). The percentage increases in apoptotic cells were 2.6, 17.6 and 29.5\% in MDA-MB-231 cells, and
2.0, 9.1 and $23.9 \%$ in MCF-7 cells at the respective silymarin concentrations (Fig. 3C and D). These results suggest that the silymarin-induced decrease in MDA-MB-231 and MCF-7 cell viability was closely associated with apoptosis.

Effects of silymarin on apoptosis-related proteins in breast cancer cells. Apoptosis occurs via various protein interactions. Among the proteins involved, the Bcl-2 family regulates the balance between pro-apoptotic and anti-apoptotic proteins (12). PARP, which resides in the nucleus is decomposed by activation of the caspase cascade, which induces apoptosis (13). Therefore, western blotting was conducted to investigate the changes in the apoptosis-related protein expression in 
A

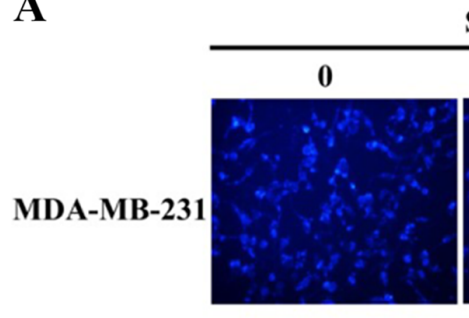

Silymarin $(\mu \mathrm{g} / \mathrm{ml})$

100

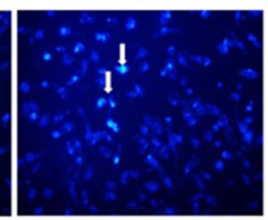

C

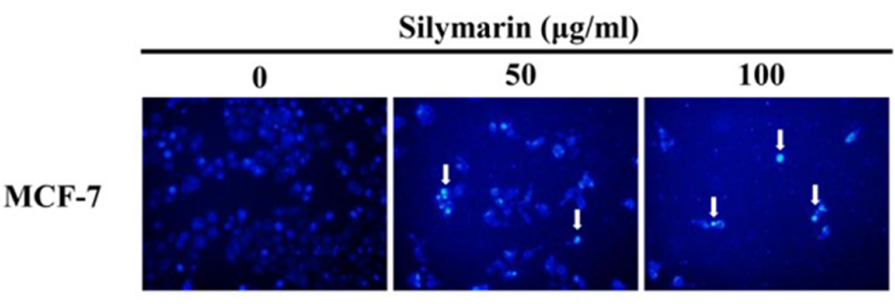

B 200

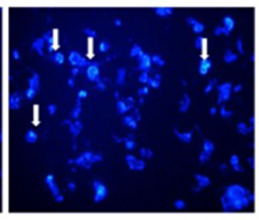

D

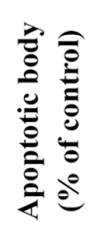

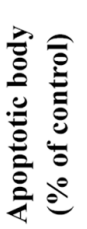

MDA-MB-231

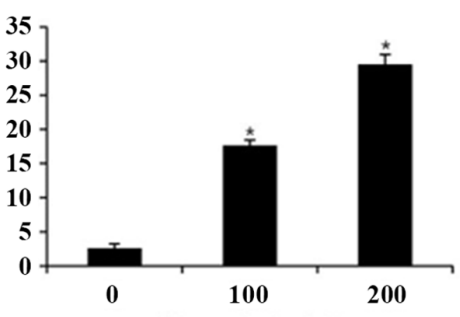

Silymarin $(\mu \mathrm{g} / \mathrm{ml})$

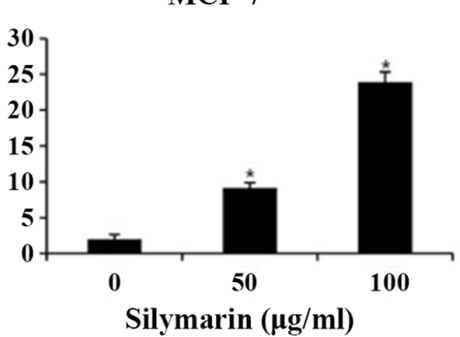

Figure 3. Effects of silymarin on breast cancer cell apoptosis. (A) MDA-MB-231 and (C) MCF-7 cells were treated with silymarin for 24 h and apoptotic bodies were stained with DAPI. Arrows indicate chromatin condensation in the MDA-MB-231 and MCF-7 cells. Apoptotic bodies were examined under a fluorescence microscope (magnification, x200). Graphs showing the quantification of apoptotic bodies in (B) MDA-MB-231 and (D) MCF-7 cells. Data are presented as the mean $\pm \mathrm{SD}$ of three independent experiments. ${ }^{*} \mathrm{P}<0.05$ vs. untreated control $(0 \mu \mathrm{g} / \mathrm{ml}$ silymarin).

silymarin-treated MDA-MB-231 and MCF-7 cells treated with silymarin. The expression levels of Bax, cleaved PARP, and cleaved caspase-9 (pro-apoptotic proteins) were increased in MDA-MB-231 and MCF-7 cells treated with silymarin, while the expression of Bcl-2 (anti-apoptotic protein) expression was decreased (Fig. 4A and B). These results exhibited that the inhibitory effects of silymarin on MDA-MB-231 and MCF-7 breast cancer cells viability were induced by modulating the expression of apoptosis-related proteins.

Effects of silymarin on MAPK signaling pathways in breast cancer cells. Various kinases of the MAPK signaling pathway, which is known as an intracellular signaling transport system, intervene in intercellular and intracellular reactions, in response to changes in the cellular environment (16). Generally, ERK1/2 promotes cell proliferation, while JNK and P38 have the opposite effect. MDA-MB-231 and MCF-7 cells were treated with different concentrations of silymarin to examine its effects on proteins of the MAPK signaling pathway. Silymarin treatment (at different concentrations for $24 \mathrm{~h}$ ) resulted in the increased expression of p-JNK, while p-ERK1/2 and p-P38 were decreased in both MDA-MB-231 and MCF-7 cells (Fig. 5A and B). These results confirm that silymarin-induced apoptosis in MDA-MB-231 and MCF-7 breast cancer cells was related with the modulation of the MAPK signaling pathway.

Effects of silymarin on breast cancer tumor growth in an in vivo animal model. Based on the results of in vitro experimentation, we performed a xenograft with MCF-7 breast cancer cells. MCF-7 breast cancer cells were implanted into the hypodermis of five-week-old male BALB/c nude mice., and the effects of silymarin on MCF-7 tumor growth were assessed. Silymarin was diluted in PBS and was orally administered at 25 or $50 \mathrm{mg} / \mathrm{kg}$ five times per week for 3 weeks; the tumor size was measured twice per week. Compared with the control group, at 21 days post drug administration, the tumor in administered with silymarin were smaller than those of the control group (Fig. 6A). Compared with the control group in 21 days, tumors in the 25 and $50 \mathrm{mg} / \mathrm{kg}$ silymarin-treated groups were 62.3 and $38.0 \%$ smaller (Table I). The tumor weight was $0.46 \pm 0.12 \mathrm{~g}$ in the control group, $0.19 \pm 0.11 \mathrm{~g}$ in the $25 \mathrm{mg} / \mathrm{kg}$ silymarin-treated group and $0.30 \pm 0.13 \mathrm{~g}$ in the $50 \mathrm{mg} / \mathrm{kg}$ silymarin-treated group (Fig. 6B). These findings suggest that silymarin has an inhibitory effect on MCF-7 tumor growth, while the body weights of the control and silymarin-administered groups did not alter significantly across the 21 day period (Fig. 6C).

Effects of silymarin on the apoptosis in breast cancer tumor tissues. A TUNEL assay was performed to determine whether the inhibitory effects of silymarin on MCF-7 tumors growth, were caused by apoptosis. As a result, the number of TUNEL-positive cells was increased in the group administered with $25 \mathrm{mg} / \mathrm{kg}$ silymarin compared with that of the control group (Fig. 6D). Compared with the control group, the group administered with $25 \mathrm{mg} / \mathrm{kg}$ silymarin witnessed a $48 \%$ increase in TUNEL-positive cells (Fig. 6E). These findings indicate that following silymarin treatment, DNA segmentation in MCF-7 tumors of nude mice resulted from apoptosis, and that silymarin induced apoptosis in the MCF-7 tumors.

Effects of silymarin on MAPK signaling pathways in breast cancer tumor tissues. In response to cellular environmental changes, the MAPK signaling pathway, which is known as an intracellular signaling transport system, intervene in intercellular and intracellular reactions. ERK1/2 is an important modulator of various biological activities that involve cell survival and proliferation (16). In the present study, silymarin 

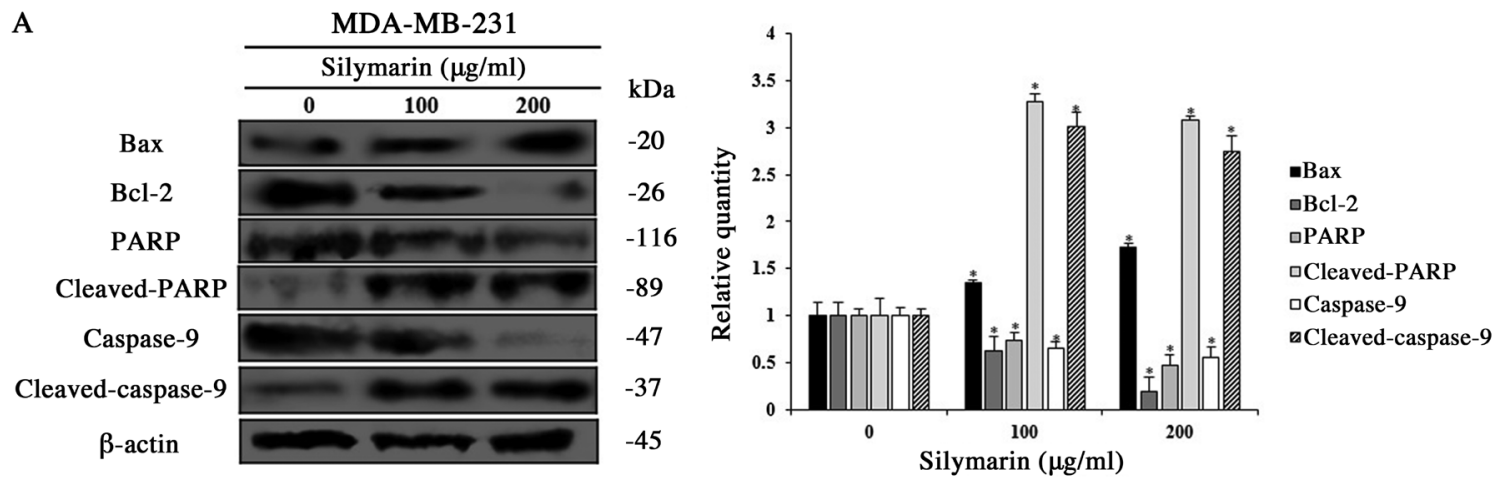

B
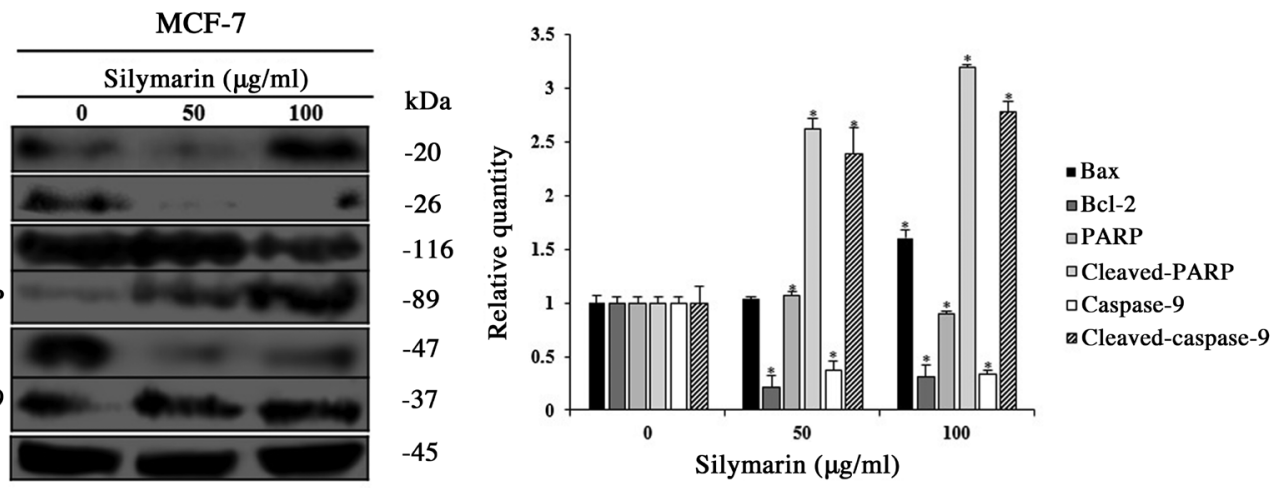

Figure 4. Effects of silymarin on the expression levels of apoptosis-related proteins in breast cancer cells. (A) MDA-MB-231 and (B) MCF-7 cells were treated with silymarin for $24 \mathrm{~h}$, and cells were harvested and the protein expression levels of Bcl-2, Bax, cleaved PARP and caspase- 9 were assessed by western blotting. Equal protein loading was confirmed by the analysis of $\beta$-actin expression. Data are presented as the mean $\pm \mathrm{SD}$ of three independent experiments. ${ }^{*} \mathrm{P}<0.05$ vs. untreated control $(0 \mu \mathrm{g} / \mathrm{ml}$ silymarin). PARP, poly (ADP-ribose) polymerase.

A

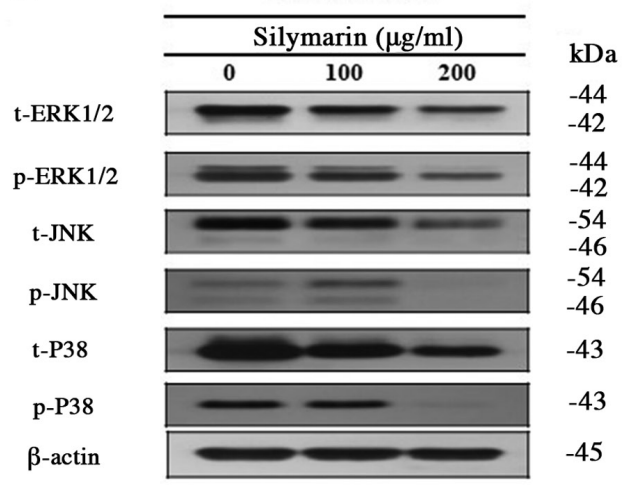

B

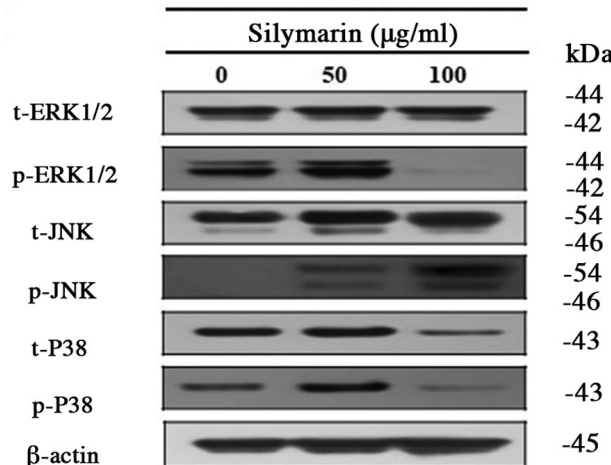

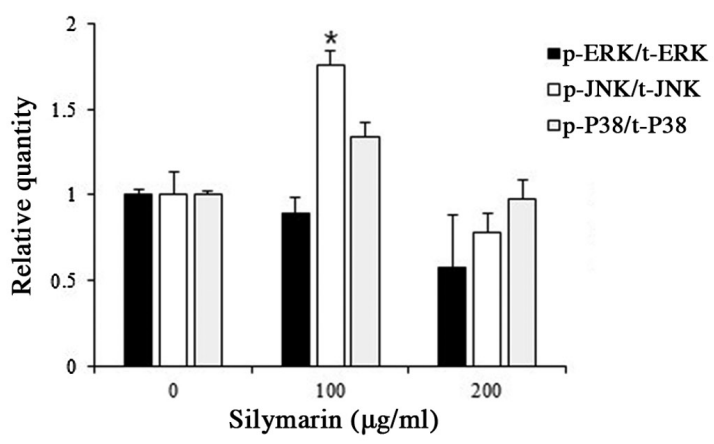

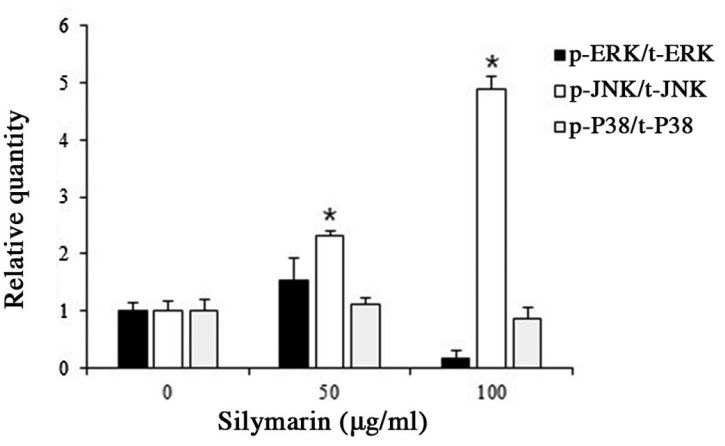

Figure 5. Effect of silymarin on the activation of the MAPK signaling pathway in MDA-MB-231 and MCF-7 cells. To determine the expression levels of MAPK signaling pathway proteins, (A) MDA-MB-231 and (B) MCF-7 cells were treated with silymarin for $24 \mathrm{~h}$, and western blot analysis was performed using antibodies against ERK1/2, p-ERK1/2, JNK, p-JNK, P38 and p-P38. Extracted proteins were resolved by SDS-PAGE. Data are presented as the mean \pm SD of three independent experiments. " $\mathrm{P}<0.05$ vs. untreated control ( $0 \mu \mathrm{g} / \mathrm{ml}$ silymarin). MAPK, mitogen-activated protein kinase; p-, phosphorylated; t-, total. 
A

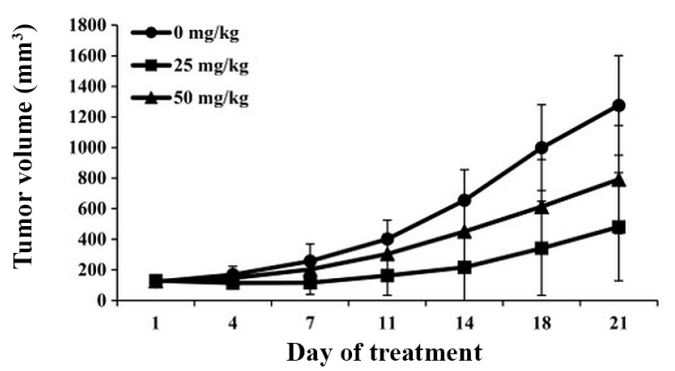

D
B

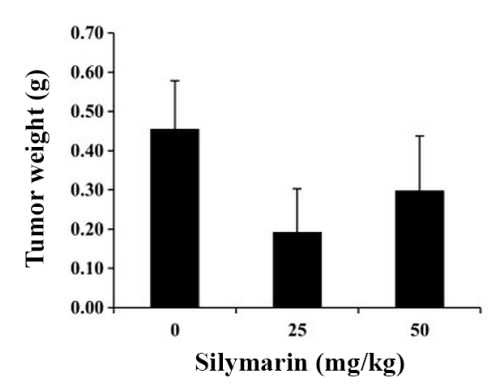

C

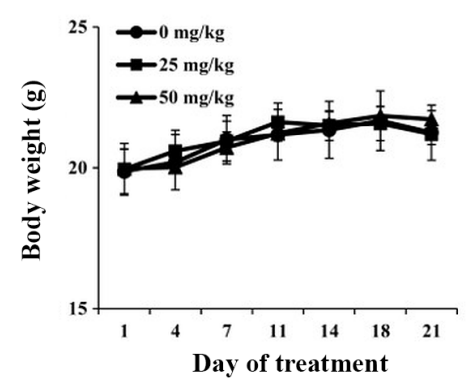

E
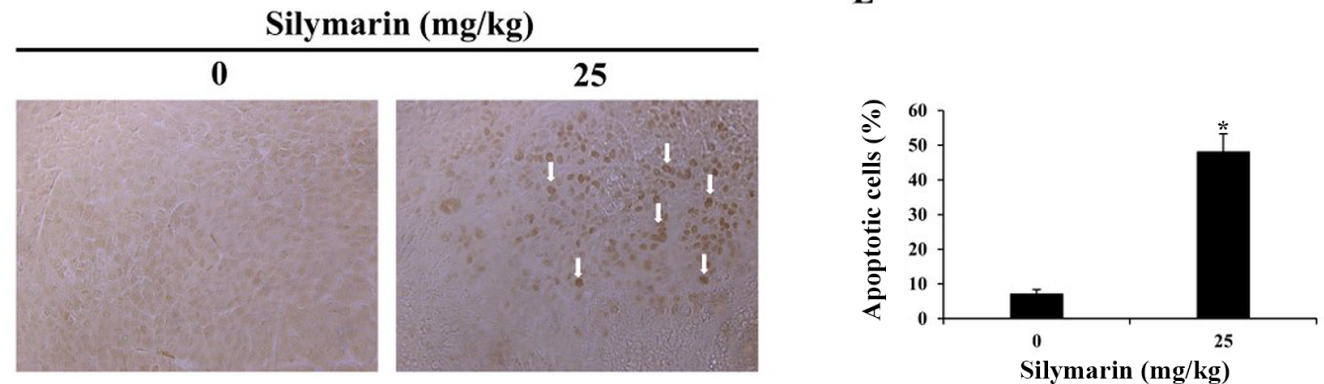

Figure 6. Effects of silymarin on tumor growth and apoptosis in MCF-7 tumor tissues. Nude mice xenograft models using the MCF-7 tumors were treated with silymarin for 21 days, and (A) tumor volume and (B) tumor weight were determined. And (C) nude mice body weight was measured. Data are presented as the mean \pm SEM. (D) Arrows indicate apoptosis, which was measured in tumor tissues using a TUNEL assay. (E) Graphs presenting the quantification of TUNEL-positive cells. Slides were observed under a microscope (magnification, $\mathrm{x} 400)$. ${ }^{*} \mathrm{P}<0.05$ vs. untreated control $(0 \mu \mathrm{g} / \mathrm{ml}$ silymarin).

A

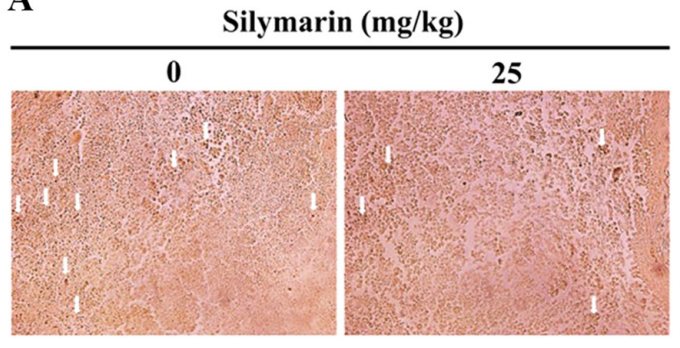

B

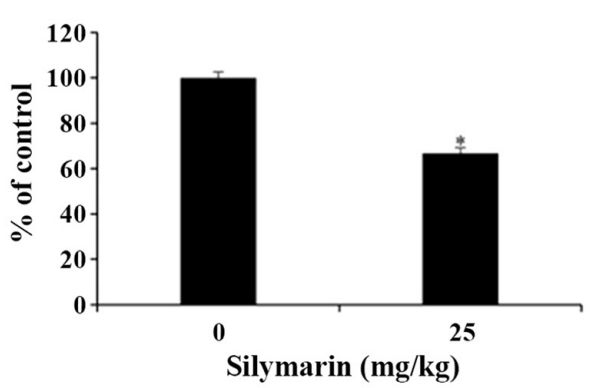

Figure 7. Effects of silymarin on p-ERK1/2 expression in MCF-7 tumor tissues. (A) BALB/c nude mice were maintained under a 12-h light/dark cycle, and housed at controlled temperature $\left(23 \pm 3^{\circ} \mathrm{C}\right)$ and humidity $(40 \pm 10 \%)$ conditions. MCF-7 cells were injected subcutaneously into the right flank of donor nude mice, and silymarin was orally administrated 5 times per week at a dose of $25 \mathrm{mg} / \mathrm{kg}$ body weight for 3 weeks. Immunohistochemistry was conducted using antibodies against p-ERK (magnification, x200). Arrows indicate p-ERK1/2-positive staining. (B) Graph presenting the quantification of p-ERK1/2 positive staining. "P<0.05 vs. untreated control ( $0 \mu \mathrm{g} / \mathrm{ml}$ silymarin). p-, phosphorylated.

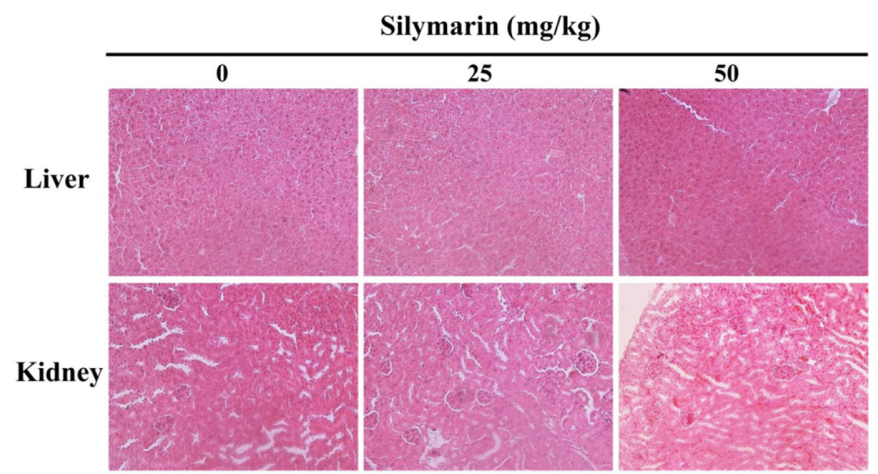

Figure 8. Histological observations in silymarin-treated nude mice. At 21 days post-silymarin treatment, the mice were sacrificed and the livers from mice in each group were removed and processed for histopathological analysis (hematoxylin and eosin staining) and observed using a microscope (magnification, x200). was administered to nude mice with xenograft tumors, and then immunohistochemistry was used to confirm the expression of p-ERK1/2 in the MCF-7 tumor tissues. As a result, the $25 \mathrm{mg} / \mathrm{kg}$ silymarin-treated group revealed the decrease in expression of p-ERK1/2 in comparison with the control group (Fig. 7A). Also, the quantitative expression of p-ERK1/2 decreased from $100 \%$ in the control group to $66.75 \%$ in the silymarin-treated group (Fig. 7B).

Silymarin-induced histopathological changes in liver and kidney tissues. To investigate the level of silymarin-associated organ toxicity, histological changes in the liver and kidney tissues of MCF-7 tumor xenografted mice were confirmed by hematoxylin and eosin staining (Fig. 8). The results confirmed that histopathological abnormalities were not exhibited, therefore demonstrating that the apoptotic effect of silymarin on 
Table I. Tumor inhibition rate of xenograft mice with MCF-7 tumors treated with silymarin.

\begin{tabular}{lccc}
\hline $\begin{array}{l}\text { Silymarin, } \\
\mathrm{mg} / \mathrm{kg}\end{array}$ & $\begin{array}{r}\text { Pre-experiment } \\
\text { size, } \mathrm{mm}^{3}\end{array}$ & $\begin{array}{c}\text { Post-experiment } \\
\text { size, } \mathrm{mm}^{3}\end{array}$ & $\begin{array}{c}\text { Inhibition } \\
\text { rate }^{\mathrm{b}}, \%\end{array}$ \\
\hline $0^{\mathrm{a}}$ & 122.0 & 1275.4 & \\
25 & 129.5 & 481.3 & 62.3 \\
50 & 126.5 & 790.5 & 38.0 \\
\hline
\end{tabular}

${ }^{\mathrm{a}}$ Control. ${ }^{\mathrm{b}}$ Data are presented as percentages relative to the control.

human breast cancer cells has no histological side-effects in a mice xenograft model.

\section{Discussion}

Owing to developments in modern medicine, the average life expectancy has increased. However, due to changes in diet, the rate of cancer occurrence and death rates of cancer have also increased. Subsequently, interest in substances for cancer prophylaxis and treatment is rising (18). Breast cancer is the most common type of cancer in women worldwide, with continuously increasing rates of occurrence and death. Following early detection, breast cancer treatment can increase patient the survival rates of patients, but is accompanied by many side-effects (19). Many studies are focused on the anticancer and immunoregulatory effects of natural materials without such side-effects. Among these compounds, silymarin has been used in various developed counties, including European nations to protect the livers of patients with chronic epilepsy. Silymarin is a natural substance of the Composittae family obtained from the fruit and seeds of thistles (Silybum marianus L.). Recent studies have reported that silymarin is used as a healthy functional food in recognition of the hepatoprotective effects and has been reported the various effects such as inflammation $(750 \mathrm{mg} / \mathrm{kg} / \mathrm{day})$, antioxidants $\left(150 \mathrm{mg} / \mathrm{kg}^{-1}\right)$ and anti-cancer $(25,50 \mathrm{mg} / \mathrm{kg})(6-10)$. In present study, the apoptosis induction and pharmacological mechanisms of silymarin were investigated using MDA-MB-231 and MCF-7 breast cancer cells, and the inhibitory effects on tumor growth were verified the in vivo.

To investigate the effects of silymarin on MDA-MB-231 and MCF-7 breast cancer cells, the MTT assay was used to assess the cell viability. After treatment with silymarin for $24 \mathrm{~h}$, a significant concentration-dependent decrease in the cell viability rate, was observed, which was evident from $75 \mu \mathrm{g} / \mathrm{ml}$ in MDA-MB-231 cells and $50 \mu \mathrm{g} / \mathrm{ml}$ in MCF-7 cells. The wound healing assay showed that the proliferation of the MDA-MB-231 and MCF-7 breast cancer cells was also inhibited depending on the concentration. Fan et al (20) reported a concentration-dependent decrease in ovarian cancer cells treated with different silymarin concentrations, where the effect was observed from $50 \mu \mathrm{g} / \mathrm{ml}$. Deep et al (10) observed a concentration- and time-dependent decrease in the cell viability rate of PC-3 prostate cancer cells when treated with 50 and $100 \mu \mathrm{g} / \mathrm{ml}$ silymarin. Additionally, Kalla et al (21) demonstrated that the viability and proliferation of breast cancer cells were inhibited by different concentrations of silymarin. Vaid et al (22) also observed that the viability rates of melanoma cells were inhibited in a concentration-dependent manner when treated with different concentrations of silymarin. These findings support that silymarin inhibits the viability rate and the proliferation of MDA-MB-231 and MCF-7 breast cancer cells. MCF-7 cells were also found to be more sensitive to silymarin than MDA-MB-231. However, the underlying reason for those differences in sensitivity, requires further study.

When apoptosis is induced, the apoptotic bodies appear within the cell and nuclear condensation and DNA fragmentation occur (11). In the present study, DAPI staining was performed to confirm whether the effects of silymarin on breast cancer cells were caused by apoptosis. When breast cancer cells were treated with silymarin for $24 \mathrm{~h}$, MDA-MB-231 cells at 0,100 or $200 \mu \mathrm{g} / \mathrm{ml}$ and MCF-7 cells at 0,50 or $100 \mu \mathrm{g} / \mathrm{ml}$, chromosomal condensation and an increase in apoptotic bodies (hallmark characteristics of apoptosis) were observed in both cell lines. In addition, to quantify the degree of apoptotic induction, DAPI-positive cells were also quantified. At the corresponding concentrations, 2.6, 17.6 and 29.5\% of MDA-MB-231, and 2.0, 9.1 and 23.9\% of MCF-7 cells exhibited DAPI staining, demonstrating a concentration-dependent increase in DAPI-positive cells, compared with the control group. In support of these findings, Fan et al (20) reported apoptotic bodies in ovarian cancer cells treated with different concentrations of silymarin. Furthermore, Katiyar et al (23) revealed that apoptotic bodies in the skin epidermal cells were increased in a concentration-dependent manner following silymarin treatment. To summarize, the decrease in the viability of silymarin-treated MDA-MB-231 and MCF-7 breast cancer cells was closely related with the induction of apoptosis.

Various bioactivities are required to induce apoptosis. Among these, the Bcl-2 family exists in the equilibrium with pro-apoptotic proteins and anti-apoptotic proteins. When this equilibrium is disrupted, apoptosis is induced (12). Therefore, western blotting was performed to observe silymarin-induced changes in the expression levels of apoptosis-related proteins in MDA-MB-231 and MCF-7 breast cancer cells. As a result, expression of the pro-apoptotic protein Bax was increased in MDA-MB-231 and MCF-7 cells, while anti-apoptotic Bcl-2 expression was decreased. The expression levels of cleaved caspase-9 and cleaved PARP also were increased in both breast cancer cells. Katiyar et al (23) reported that in the skin epidermal cells, the expression levels of Bax and cleaved PARP were increased, while Bcl-2 expression was decreased following treatment with different concentrations of silymarin. Fan et al (20) also found that the expression levels of Bax and cleaved caspase- 9 in ovarian cancer cells were increased in a concentration-dependent manner when treated with silymarin, while Bcl-2 expression was decreased in the same manner. These findings demonstrate that silymarin regulates the expression levels of apoptosis proteins in MDA-MB-231 and MCF-7 breast cancer cells and that silymarin-associated inhibitory effects on breast cancer cell viability and proliferation were induced by apoptosis.

The MAPK signaling pathways are known to regulators of cell proliferation, apoptosis, proliferation, and differentiation. Several types of MAPK signaling pathways including those of 
ERK1/2, P38 MAPK, JNK/SAPK (16). In the current study, the expression levels of ERK1/2, P38, and JNK were investigated in silymarin-treated MDA-MB-231 and MCF-7 breast cancer cells. When treated with different concentration of silymarin, p-ERK1/2 and p-P38 expression levels were decreased, while p-JNK expression levels were increased in both cell lines. Li et al (24) reported a concentration-dependent increase in the p-P38 and p-ERK1/2 expressions in A375-S2 melanoma cells treated with different silymarin concentrations. Furthermore, Huang et al (25) observed that p-P38 and p-JNK expression levels were regulated in a concentration-dependent manner when HeLa uterine cervical cancer cells were treated with different concentration of silymarin. Also, Pereira et al (26) observed that P38 MAPK inhibition results in ROS upregulation, which in turn activates the JNK pathway via the inactivation of phosphatases, sensitizing human tumor cells to induced apoptosis. Recent, the natural compound in plants such as flavonoids was reported that the effect of anti-cancer in breast cancer, including MDA-MB-231 and MCF-7. Also, these effect of anti-cancer in flavonoids induced the apoptosis through the various caspase, including caspase- 3 and caspase- 9 that was closely related with activation of MAPK signaling pathway such as, ERK and JNK in MDA-MB-231 (27-29) and MCF-7 (30,31). These findings suggest that silymarin induces apoptosis by the modulating of the MAPK signaling pathways in MDA-MB-231 and MCF-7 breast cancer cells. Based on these results, silymarin was used to induce apoptosis in these cells, in order to inhibit cell growth and proliferation in vitro. MCF-7 cells were also found to be more sensitive to silymarin treatment than for MDA-MB-231 cells. But, to determine the relation of between caspases and MAPK signaling pathway that was main mechanism as the activation of MAPK signaling pathway including ERK JNK and P38, we have to study more. Also, we should study more to confirm the phospho-JNK mediated pathways in MDA-MB-231 and MCF-7 cells are possibly distinct and related to caspase- 9 cascade in both cases.

As a result of these findings, the MCF-7 cells were transplanted into nude mice, and silymarin was administered to observe the effects on MCF-7 tumors growth. Following oral administration of silymarin at 0,25 or $50 \mathrm{mg} / \mathrm{kg}$ for 3 weeks, the tumor size began to decrease in comparison to in the control group from 7 days post-administration. By the 21 day, tumor growth was inhibited by $62.3 \%$ in the $25 \mathrm{mg} / \mathrm{kg}$ silymarin-treated group and $38.0 \%$ in the $50 \mathrm{mg} / \mathrm{kg}$ group. The tumor weights were $0.46 \mathrm{~g}$ in the control group, $0.19 \mathrm{~g}$ in the $25 \mathrm{mg} / \mathrm{kg}$ silymarin-administered group and $0.30 \mathrm{~g}$ in the $50 \mathrm{mg} / \mathrm{kg}$ silymarin-administered group, revealing a downward trend in tumor size in the silymarin-administered groups. Wu et al (9) found that tumor expansion was inhibited in a concentration-dependent manner in lung cancer tissues following the intraperitoneal injection of $25 \mathrm{mg} / \mathrm{kg}$ or $50 \mathrm{mg} / \mathrm{kg}$ of silymarin. Also, Singh et al (32) is reported a decrease in A431 epidermal cancer cell tumor growth in a group injected with silymarin for 5 weeks, compared with that in the control group. These findings support that silymarin inhibits the growth of MCF-7 tumors.

The TUNEL assay was performed to confirm whether the inhibition of tumor growth was caused by apoptosis induction. As a result, TUNEL-positive apoptotic cells were increased by $48.0 \%$ in the groups administered with $25 \mathrm{mg} / \mathrm{kg}$ of silymarin, respectively. Singh et al (32) reported that cells positive for apoptosis bodies were increased in a group of epidermal cancer cell xenografted mice with epidermal cancer cells treated with silymarin compared with those in the control group. These results demonstrate that DNA fragmentation in the nucleus was due to apoptosis, and that silymarin inhibits the MCF-7 tumor growth via apoptotic induction.

ERK is as an important regulator of apoptosis, with known roles in cell proliferation and differentiation (16). After the administration of silymarin to xenograft mice, immunohistochemistry was performed to observe ERK1/2 activities in MCF-7 tumor tissues. As a result, the level of p-ERK1/2 expression was decreased in the $25 \mathrm{mg} / \mathrm{kg}$ silymarin-treated group compared with those in the control group, reflecting the same results obtained from the western blotting in vitro.

Histopathological studies confirmed that silymarin treatment does not result in obvious lesions of the liver and kidney. In addition, body weight observations indicated no signs of systemic toxicity. To the best of our knowledge, the in vivo results of the present study demonstrate the apoptotic effects of silymarin in human breast cancer are not associated with side-effects, as has been previously indicated $(33,34)$. However, we have to study more for the lack of assessment of apoptosis and toxicity in healthy tissues following silymarin treatment as a potential limitation to the experiments.

To summarize, the findings of the present study indicate that breast tumor growth was inhibited by silymarin as the result of apoptosis induction was the cause of such inhibition, suggesting that silymarin inhibits the growth of breast cancer cells both in vitro and in vivo. Taken together, these results illustrate a novel effect of silymarin and demonstrate its potentially chemotherapeutic use in patient with breast cancer.

\section{Acknowledgements}

Not applicable.

\section{Funding}

This research was supported by the Basic Science Research Program through the National Research Foundation of Korea (NRF) funded by the Ministry of Education, Science and Technology (NRF 2017R1A2B4005516).

\section{Availability of data and materials}

The datasets used and/or analyzed during the current study are available from the corresponding author on reasonable request.

\section{Authors' contributions}

SHK, GSC, HJK and JYJ designed the experiments to perform, and then wrote the manuscript. SHK, GSC, ESY, JSW, JHL, SHH and SHJ performed the experiments. SHK, GSC and ESY analyzed and interpreted the results. GSC performed the statistical analysis. ESY and JSW assembled the data. JYJ contributed to every process as a supervisor. SHK, GSC, ESY, JSW, JHL, SHH and JYJ assessed and confirmed the authenticity of all the raw data. All authors read and approved the final manuscript. 


\section{Ethics approval and consent to participate}

The animal experiments were conducted in accordance with the regulations of the Institutional Animal Care and Use Committee with the approval of the Ethics Committee of Kongju National University (Yesan, South Korea).

\section{Patient consent for publication}

Not applicable.

\section{Competing interests}

The authors declare that they have no competing interests.

\section{References}

1. Liu MM, Huang Y and Wang J: Developing phytoestrogens for breast cancer prevention. Anticancer Agents Med Chem 12: 1306-1313, 2012

2. Li Z, Qin B, Qi X, Mao J and Wu D: Isoalantolactone induces apoptosis in human breast cancer cells via ROS-mediated mitochondrial pathway and downregulation of SIRT1. Arch Pharm Res 39: 1441-1453, 2016.

3. Nizamutdinova IT, Lee GW, Son KH, Jeon SJ, Kang SS, Kim YS Lee JH, Seo HG, Chang KC and Kim HJ: Tanshinone I effectively induces apoptosis in estrogen receptor-positive (MCF-7) and estrogen receptor-negative (MDA-MB-231) breast cancer cells. Int J Oncol 33: 485-491, 2008.

4. Panche AN, Diwan AD and Chandra SR: Flavonoids: An overview. J Nutr Sci 5: e47, 2016.

5. Comelli MC, Mengs U, Schneider C and Prosdocimi M: Toward the definition of the mechanism of action of silymarin: Activities related to cellular protection from toxic damage induced by chemotherapy. Integr Cancer Ther 6: 120-129, 2007.

6. Chen IS, Chen YC, Chou CH, Chuang RF, Sheen LY and Chiu CH: Hepatoprotection of silymarin against thioacetamide-induced chronic liver fibrosis. J Sci Food Agric 92: 1441-1447, 2012.

7. Sherif IO and Al-Gayyar MM: Antioxidant, anti-inflammatory and hepatoprotective effects of silymarin on hepatic dysfunction induced by sodium nitrite. Eur Cytokine Netw 24: 114-121, 2013.

8. El-Lakkany NM, Hammam OA, El-Maadawy WH, Badawy AA Ain-Shoka AA and EbeidFA;EI-LakkanyNM: Anti-inflammatory/ anti-fibrotic effects of the hepatoprotective silymarin and the schistosomicide praziquantel against Schistosoma mansoni-induced liver fibrosis. Parasit Vectors 5: 9, 2012.

9. Wu T, Liu W, Guo W and Zhu X: Silymarin suppressed lung cancer growth in mice via inhibiting myeloid-derived suppressor cells. Biomed Pharmacother 81: 460-467, 2016.

10. Deep G, Singh RP, Agarwal C, Kroll DJ and Agarwal R: Silymarin and silibinin cause G1 and G2-M cell cycle arrest via distinct circuitries in human prostate cancer PC 3 cells: A comparison of flavanone silibinin with flavanolignan mixture silymarin. Oncogene 25: 1053-1069, 2006.

11. Han SI, Kim YS and Kim TH: Role of apoptotic and necrotic cell death under physiologic conditions. BMB Rep 41: 1-10, 2008.

12. Oda E, Ohki R, Murasawa H, Nemoto J, Shibue T, Yamashita T, Tokino T, Taniguchi T and Tanaka N: Noxa, a BH3-only member of the Bcl-2 family and candidate mediator of p53-induced apoptosis. Science 288: 1053-1058, 2000.

13. Donovan M and Cotter TG: Control of mitochondrial integrity by Bcl-2 family members and caspase-independent cell death Biochim Biophys Acta 1644: 133-147, 2004.

14. Oltvai ZN, Milliman CL and Korsmeyer SJ: Bcl-2 heterodimerizes in vivo with a conserved homolog, Bax, that accelerates programmed cell death. Cell 74: 609-619, 1993.

15. Brentnall M, Rodriguez-Menocal L, De Guevara RL, Cepero E and Boise LH: Caspase-9, caspase-3 and caspase-7 have distinct roles during intrinsic apoptosis. BMC Cell Biol 14: 32, 2013.
16. Yang Y,Zhu X, Chen Y,Wang X and Chen R: p38 and JNK MAPK, but not ERK1/2 MAPK, play important role in colchicine-induced cortical neurons apoptosis. Eur J Pharmacol 576: 26-33, 2007.

17. Joneson T, White MA, Wigler MH and Bar-Sagi D: Stimulation of membrane ruffling and MAP kinase activation by distinct effectors of RAS. Science 271: 810-812, 1996.

18. Doll R: The lessons of life: Keynote address to the nutrition and cancer conference. Cancer Res 52 (Suppl 7): 2024s-2029s, 1992.

19. Porter PL: Global trends in breast cancer incidence and mortality. Salud Publica Mex 51 (Suppl 2): s141-s146, 2009.

20. Fan L, Ma Y, Liu Y, Zheng D and Huang G: Silymarin induces cell cycle arrest and apoptosis in ovarian cancer cells. Eur J Pharmacol 743: 79-88, 2014.

21. Kalla PK, Chitti S, Aghamirzaei ST, Senthilkumar R and Arjunan S: Anti-cancer activity of silymarin on MCF-7 and NCIH-23 cell lines. Adv Biol Res (Faisalabad) 8: 57-61, 2014.

22. Vaid M, Singh T, Prasad R and Katiyar SK: Silymarin inhibits melanoma cell growth both in vitro and in vivo by targeting cell cycle regulators, angiogenic biomarkers and induction of apoptosis. Mol Carcinog 54: 1328-1339, 2015.

23. Katiyar SK, Roy AM and Baliga MS: Silymarin induces apoptosis primarily through a p53-dependent pathway involving $\mathrm{Bcl}-2 / \mathrm{Bax}$, cytochrome $\mathrm{c}$ release, and caspase activation. Mol Cancer Ther 4: 207-216, 2005.

24. Li LH, Wu LJ, Tashiro SI, Onodera S, Uchiumi F and Ikejima T: The roles of Akt and MAPK family members in silymarin's protection against UV-induced A375-S2 cell apoptosis. Int Immunopharmacol 6: 190-197, 2006.

25. Huang Q, Wu LJ, Tashiro S, Onodera S, Li LH and Ikejima T: Silymarin augments human cervical cancer HeLa cell apoptosis via P38/JNK MAPK pathways in serum-free medium. J Asian Nat Prod Res 7: 701-709, 2005.

26. Pereira L,Igea A, Canovas B, Dolado I and Nebreda AR: Inhibition of p38 MAPK sensitizes tumour cells to cisplatin-induced apoptosis mediated by reactive oxygen species and JNK. EMBO Mol Med 5: 1759-1774, 2013.

27. Cui L, Bu W, Song J, Feng L, Xu T, Liu D, Ding W, Wang J, Li C, $\mathrm{Ma} \mathrm{B}$, et al: Apoptosis induction by alantolactone in breast cancer MDA-MB-231 cells through reactive oxygen species-mediated mitochondrion-dependent pathway. Arch Pharm Res 41: 299-313, 2018.

28. Lin KL, Su JC, Chien CM, Tseng CH, Chen YL, Chang LS and Lin SR: Naphtho[1,2-b]furan-4,5-dione induces apoptosis and S-phase arrest of MDA-MB-231 cells through JNK and ERK signaling activation. Toxicol In Vitro 24: 61-70, 2010.

29. Yu P, Zhang C, Gao CY, Ma T, Zhang H, Zhou MM, Yang YW, Yang L and Kong LY: Anti-proliferation of triple-negative breast cancer cells with physagulide P: ROS/JNK signaling pathway induces apoptosis and autophagic cell death. Oncotarget 8: 64032-64049, 2017.

30. Palit S, Kar S, Sharma G and Das PK: Hesperetin induces apoptosis in breast carcinoma by triggering accumulation of ROS and activation of ASK1/JNK pathway. J Cell Physiol 230: $1729-1739,2015$

31. Rabi T and Banerjee S: Novel synthetic triterpenoid methyl 25-h ydroxy-3-oxoolean-12-en-28-oate induces apoptosis through JNK and p38 MAPK pathways in human breast adenocarcinoma MCF-7 cells. Mol Carcinog 47: 415-423, 2008.

32. Singh RP, Tyagi AK, Zhao J and Agarwal R: Silymarin inhibits growth and causes regression of established skin tumors in SENCAR mice via modulation of mitogen-activated protein kinases and induction of apoptosis. Carcinogenesis 23: 499-510, 2002.

33. Kim LH, Khadka S, Shin JA, Jung JY, Ryu MH, Yu HJ, Lee HN, Jang B, Yang IH, Won DH, et al: Nitidine chloride acts as an apoptosis inducer in human oral cancer cells and a nude mouse xenograft model via inhibition of STAT3. Oncotarget 8: 91306-91315, 2017.

34. Shivakumar P, Rani MU, Reddy AG and Anjaneyulu Y: A study on the toxic effects of Doxorubicin on the histology of certain organs. Toxicol Int 19: 241-244, 2012.

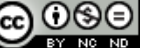

This work is licensed under a Creative Commons Attribution-NonCommercial-NoDerivatives 4.0 International (CC BY-NC-ND 4.0) License. 